\title{
Magneto-seismology: effect of inhomogeneous magnetic field on transversal coronal loop oscillations
}

\author{
G. Verth \\ $\mathrm{SP}^{2} \mathrm{RC}$, Department of Applied Mathematics, University of Sheffield, Sheffield S3 7RH, UK \\ email: G.Verth@sheffield.ac.uk
}

\begin{abstract}
The extreme-ultraviolet (EUV) imagers onboard the planned Solar Dynamics Observatory $(S D O)$ and Solar Orbiter $(S O)$ will offer us the best chance yet of using observations of post-flare loop oscillations to probe the fine structure of the corona. Recently developed magnetohydrodynamic (MHD) wave theory has shown that the properties of loop oscillations depend on their plasma fine structure. Up to this point, many studies have concentrated solely on the effect of plasma density stratification on coronal loop oscillations. In this paper we develop MHD wave theory which models the effect of an inhomogeneous magnetic field on coronal loop oscillations. The results have the potential to be used in testing the efficacy of photospheric magnetic field extrapolations and have important implications regarding magneto-seismology of the corona.
\end{abstract}

Keywords. (magnetohydrodynamics:) MHD, waves, magnetic fields, Sun: oscillations, Sun: magnetic fields, Sun: fundamental parameters, Sun: corona

\section{Introduction}

The next decade will be an exciting time for observing the upper solar atmosphere with new space based missions such as Hinode, Solar TErresrial Relations Observatory (STEREO), Solar Dynamics Observatory (SDO), Solar Orbiter (SO) and ground based projects such as the Advanced Technology Solar Telescope (ATST) and Frequency Agile Solar Radiotelescope (FASR). These instruments will give observers an opportunity to directly measure crucial coronal plasma parameters such as magnetic field strength and plasma density with more accuracy than ever before. However, to do this one must also have additional information regarding elemental abundances and at which height and temperature in the solar atmosphere spectral lines are formed. Unfortunately, the values for these latter physical quantities may have large uncertainties. Furthermore, methods employed to make direct measurements, ideally need to have simultaneous observations from different lines of sight. Unfortunately for all the new missions (apart from STEREO), this will not be possible. Therefore we will still need alternative methods to verify the accuracy of direct measurements. An obvious way forward is to compare the predictions of theoretical models with the observables. This also has the advantage of testing theories for completeness and correctness.

Magneto-seismology will be an invaluable tool in this regard (see the most recent reviews by Erdélyi 2006a,b and Banerjee et al. 2007). Recently developed magnetohydrodynamic (MHD) wave theory in this field has shown that the plasma fine structure of coronal loops determines their oscillatory response to solar flares. Hence, if observers estimate that an active region coronal loop has a particular magnetic structure and plasma density distribution through direct measurement and this is consistent with the oscillatory response of that loop to a flare, then this will provide strong evidence that we have 
accurately measured the fundamental plasma parameters and that our theory models the process very well (see reviews by De Pontieu \& Erdélyi 2006 and Erdélyi 2006a,b).

Any inconsistency may suggest that refinement is needed in our theoretical models and/or direct measurement techniques. Of course, inconsistency may even lead to the outright rejection of a theory. Either way, such a process is likely to enhance our understanding of the Sun's outer atmosphere fine structure and the interaction of magnetic fields and plasma under these conditions.

\section{MHD wave theory of post-flare transversal loop oscillations}

Post-flare transversal coronal loop oscillations have been observed many times using the high-resolution EUV imager onboard the Transition Region And Coronal Explorer (TRACE) (see e.g., Aschwanden et al. 1999a, 2002; Nakariakov et al. 1999; Verwichte et al. 2004; Jess et al. 2007). These oscillations were identified as the fundamental mode of the standing fast-kink wave from MHD wave theory developed by e.g., Edwin \& Roberts (1983). The basic theory models a coronal loop as a straight magnetic cylinder with different external and internal plasma densities, both of which are taken to be constants.

However, it is now clear from EUV observations using, e.g, EIT (Extreme-ultraviolet Imaging Telescope) onboard SOHO (SOlar and Heliospheric Observatory) and TRACE, that even in "static" active region coronal loops, the spatial and temporal behaviour of plasma is far more complex. Using emission measures, there is observational evidence that there is density stratification in coronal loops. In younger active regions there have been measurements of "super-hydrostatic" density scale heights that are up to four times higher than expected (Aschwanden et al. 2000, 2001). On the other hand, loops have been observed in older active regions that are close to hydrostatic equilibrium (Aschwanden et al. 1999b) with density scale heights that can be explained by gravitational stratification. The implications of this for coronal loop oscillations have been considered by e.g., Ofman \& Wang (2002) and Mendoza-Bricẽno et al. (2004). To complicate matters further, significant dynamical behaviour has also been observed in "static" loops , e.g., flows (Brekke et al. 1997; Winebarger et al. 2001, 2002) and cooling events (Winebarger et al. 2003; Schrijver 2001). This is an important point because background flows can cause complex interactions between MHD waves. Theoretically, the effect of steady state flows on MHD waves in a uniform magnetic slab-geometry was investigated by e.g. Nakariakov \& Roberts (1995), Tirry et al. (1998), Joarder et al. (1997). They found the dispersion relation for such steady states and also have shown the presence of negative energy waves. Joarder and Narayanan (2000), Somasundaram et al. (1999) and Narayanan (1991) generalised the slab studies to flux tubes but their derivation is valid only for limited parameters. A detailed and comprehensive derivation of steady flow effects on uniform MHD waveguides in cylindrical geometry (with stratification due to gravity ignored) can be found e.g. in Terra-Homem et al. (2003). They derived the dispersion relation for photospheric and coronal flux tubes, and determined the propagation windows that are Doppler shifted when compared to their static counterparts.

In light of the exciting observations from TRACE, much work has been done developing more realisitic theory of fast kink waves in coronal loops. E.g., models have been developed with inhomogeneous plasma density equilibria. Firstly, spatial variation of density in the radial direction has been included in the analysis leading to a change in period and damping of the MHD waves (Ruderman \& Roberts 2002; Goossens et al. 2002; Aschwanden et al. 2003; Van Doorsselaere et al. 2004; Arregui et al. 2007). Secondly, spatial variation of density in the longitudinal direction has been included in the analysis leading to changes in the ratios of the periods of the overtone modes to that of 


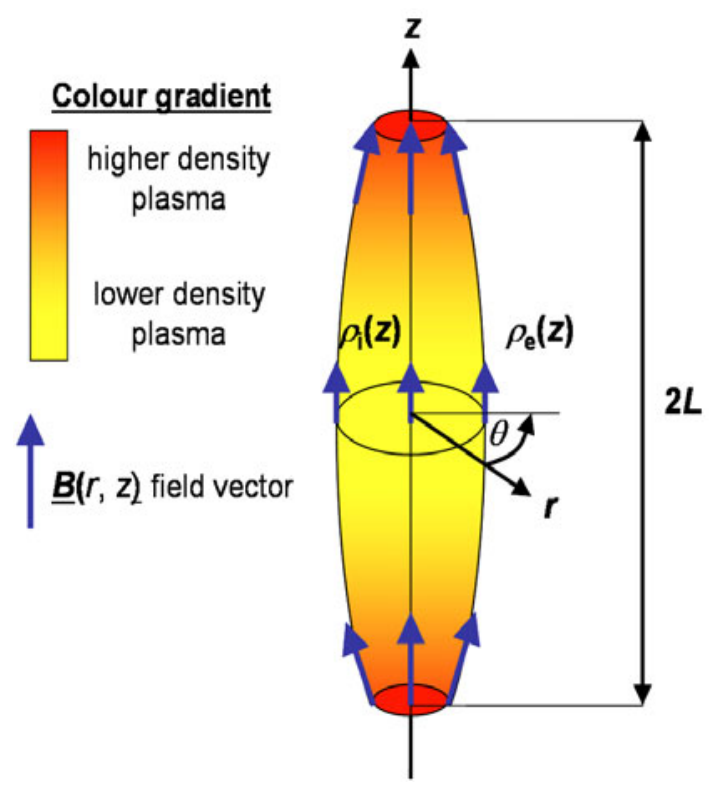

Figure 1. The equilibrium plasma density and magnetic field.

the fundamental mode and to deviations of the eigenfunctions from a single sine term in the longitudinal direction (Díaz et al. 2002; Andries et al. 2005b; Dymova \& Ruderman 2005; Arregui et al. 2006; Goossens et al. 2006; McEwan et al. 2006; Erdélyi \& Verth 2007; Verth \& Erdélyi 2007; Verth 2007; Verth et al. 2007).

To develop a more complete theory of fast kink waves coronal loops, in this paper, it is proposed to quantify the effects of both inhomogeneous plasma density and magnetic equilibria. At present, the structure of the magnetic field along coronal loops is probably even less well understood from observation than plasma density stratification (see e.g., Lopéz et al. 2006). The indirect observational evidence so far has been rather puzzling. A study of TRACE loops (Watko \& Klimchuk 2000) has shown that the cross-sectional width remains relatively constant with increasing height above the photosphere. The flux tube interpretation suggests that magnetic field is therefore almost constant along loops but this contradicts potential and force-free field extrapolations using data from the Michelson Doppler Imager (MDI) onboard SOHO, where the field lines always diverge with height. Klimchuk et al. (2000) suggested that by twisting a loop this could reduce the amount of width expansion with height. They performed a force-free extrapolation with a twisted loop embedded in a magnetic dipole and found that although the twist did reduce the expansion of the loop they could still not match the observed relatively constant thickness. It is therefore crucial that theoretical models are developed which can predict how different magnetic field structures in loops affect the properties of loop oscillations. Much work has already been done, particularly regarding the effect of magnetic twist on loop oscillations by Bennet et al. (1998), Erdélyi \& Fedun (2006, 2007) and Ruderman (2007). Furthermore, the effect of a twisted shell, i.e., tube within a tube, was studied by Erdélyi \& Carter (2006) and Carter \& Erdélyi (2007). It is hoped that models of this type that have more complex magnetically structured tubes can be tested against observations and help further advance the field of magneto-seismology. 


\section{Magnetic field and plasma density equilibrium}

Using cylindrical coordinates $(r, \theta, z)$, a magnetic flux tube of length $2 L$ is modelled with arbitrary external and internal plasma densities $\rho_{e}(z)$ and $\rho_{i}(z)$. To model a magnetic field equilibrium that decreases in strength with height above the photosphere, we construct an expanding flux tube with rotational symmetry (see Fig. 1). To do this one must have

$$
\vec{B}=B_{r}(r, z) \vec{e}_{r}+B_{z}(r, z) \vec{e}_{z}
$$

so that the solenoidal and force-free (potential) conditions are satisfied. For convenience we shall use the vector potential $\vec{A}$, defined by

$$
\vec{B}=\nabla \times \vec{A},
$$

which automatically satisfies condition solenoidal condition. To find the required form of $\vec{B}$ given by (3.1) that also satisfies the force-free condition, we define $\vec{A}$ with an azimuthal component only such that,

$$
\vec{A}=\frac{\psi(r, z)}{r} \vec{e}_{\theta}
$$

The vector potential described by (3.3) is convenient since $\psi$ is constant along field lines (see e.g. Browning \& Priest 1982). The force-free condition means that the follwing equation must be satified,

$$
\frac{\partial^{2} \psi}{\partial r^{2}}-\frac{1}{r} \frac{\partial \psi}{\partial r}+\frac{\partial^{2} \psi}{\partial z^{2}}=0
$$

Let $r_{f}$ and $r_{a}$ denote the flux tube radius at the footpoints and apex. Using the same convention as Fig. 1, it is required that the maximum $r$ and $z$ components of the magnetic field at the footpoints are

$$
B_{r}\left(r_{f}, \pm L\right)=\mp B_{r, f}
$$

and

$$
B_{z}\left(r_{f}, \pm L\right)=B_{z, f},
$$

where $B_{r, f} \geqslant 0$ and $B_{z, f}>0$. By the observations that $r_{o} \ll L$ and $B_{r} \ll B_{z}$, to a good approximation, the particular solution to Eq. (3.4) at the tube boundary $r_{o}$ is

$$
\psi\left(r_{o}, z\right) \approx \frac{B_{z, f} r_{o}^{2}}{2}\left\{1+\frac{\left(1-\Gamma^{2}\right)}{\Gamma^{2}} \frac{[\cosh (z / L)-\cosh (1)]}{1-\cosh (1)}\right\}
$$

where

$$
\Gamma=\frac{r_{a}}{r_{f}}
$$

is the expansion factor of the flux tube (see e.g, Klimchuk 2000). By Eq. (3.7), at the loop footpoints the boundary value of $\psi$ is

$$
\psi\left(r_{f}, \pm L\right) \approx \frac{B_{z, f} r_{f}^{2}}{2} .
$$

Hence using Eqs. (3.7) and (3.9) and the fact that the magnetic surface denoting the boundary of the flux tube has a constant $\psi$ value, an explicit expression is obtained for $r_{o}$ as a function of $z$ only given by 


$$
r_{o}(z) \approx r_{f}\left\{1+\frac{\left(1-\Gamma^{2}\right)}{\Gamma^{2}} \frac{\left[\cosh \left(\frac{z}{L}\right)-\cosh (1)\right]}{1-\cosh (1)}\right\}^{-\frac{1}{2}} .
$$

Similarly, expressions for $B_{r}$ and $B_{z}$ can be derived at the tube boundary as functions of $z$ only,

$$
B_{r}(z) \approx-\frac{B_{z, f}}{L} \frac{\left(1-\Gamma^{2}\right)}{2 \Gamma^{2}} \frac{\sinh \left(\frac{z}{L}\right)}{\sinh (1)} r_{o}(z)
$$

and

$$
B_{z}(z) \approx B_{z, f}\left\{1+\frac{\left(1-\Gamma^{2}\right)}{\Gamma^{2}} \frac{\left[\cosh \left(\frac{z}{L}\right)-\cosh (1)\right]}{1-\cosh (1)}\right\}
$$

\section{Linearising the MHD equations with inhomogeneous magnetic field}

The cold and ideal MHD equations are linearised by assuming small perturbations of the magnetic field, $\vec{b}=\left(b_{r}, b_{\theta}, b_{z}\right)$ about the force-free magnetic equilibrium (3.1) and velocity perturbations, $\vec{v}=\left(v_{r}, v_{\theta}, v_{z}\right)$ about a plasma in static equlilibrium. From the equilibrium magnetic field shown in Fig. 1, it is clear that perturbations normal to the surface of the tube will have both $r$ and $z$ components. The linearised MHD equations give the following relation between the $r$ and $z$ components of velocity,

$$
v_{z}=-\frac{B_{r}}{B_{z}} v_{r} .
$$

Since $B_{r} \ll B_{z}$, Eq. (4.1) shows the $z$ component of the velocity perturbation is only a small correction to the total perturbation. Therefore, in the following analysis we shall concentrate primarily on the dominant $r$ component. The equation governing the $r$ component of plasma motion is

$$
\frac{\mu \rho}{B_{z}} \frac{\partial^{2} v_{r}}{\partial t^{2}}=\frac{\partial^{2}}{\partial z^{2}}\left(\frac{B^{2}}{B_{z}} v_{r}\right)-\frac{\partial^{2} b_{z}}{\partial r \partial t}+\frac{\partial}{\partial z}\left\{\frac{B_{r}}{B_{z}}\left[\frac{\partial b_{z}}{\partial t}+\frac{1}{r} \frac{\partial}{\partial r}\left(\frac{B^{2}}{B_{z}} r v_{r}\right)\right]\right\}
$$

where $B^{2}=B_{r}^{2}+B_{z}^{2}$. It can be seen from Eq. (4.2) that the $r$ velocity component is dependent on the $z$ component of the magnetic perturbation which is governed by the following equation,

$$
\frac{\partial^{2} b_{z}}{\partial t^{2}}=-\frac{1}{r} \frac{\partial}{\partial r}\left[r \frac{B^{2}}{\mu \rho}\left(\frac{\partial b_{r}}{\partial z}-\frac{\partial b_{z}}{\partial r}\right)\right]+\frac{B_{z}}{\mu \rho} \frac{1}{r}\left[\mathcal{D}_{\|}\left(\frac{\partial}{\partial r}\left(r b_{r}\right)+r \frac{\partial b_{z}}{\partial z}\right)+\frac{\mu}{r} \frac{\partial^{2} P}{\partial \theta^{2}}\right],
$$

where operator $\mathcal{D}_{\|}$is defined as

$$
\mathcal{D}_{\|} \equiv B_{r} \frac{\partial}{\partial r}+B_{z} \frac{\partial}{\partial z}
$$

and $P$ is the total perturbation to magnetic pressure,

$$
P=\frac{\vec{B} \cdot \vec{b}}{\mu} .
$$

Eq. (4.3) contains both $z$ and $r$ magnetic field perturbations but it can be shown using observed relations that $r_{o} \ll L$ and $B_{r} \ll B_{z}$ that these are effectively decoupled. This decoupling helps simplify the mathematical analysis considerably in the following section. 


\section{Governing equation and analysis}

By Fourier analysing Eqs. (4.2) and (4.3), using the thin flux tube approximation and the fact the $B_{r} \ll B_{z}$, it can be shown that the governing equation of radial motion at the tube boundary (where all quantities can be expressed as functions of $z$ only as shown in Sect. 3) is

$$
\begin{aligned}
& \left(\frac{B_{z} v_{r}}{r_{o}^{m-1}}\right)^{\prime \prime}+\frac{m}{2 r_{o}}\left(\frac{B_{r}}{B_{z}}+4 r_{o}^{\prime}\right)\left(\frac{B_{z} v_{r}}{r_{o}^{m-1}}\right)^{\prime} \\
& \quad+\left\{\left(\frac{\omega}{c_{k}}\right)^{2}+\frac{m}{2 r_{o}}\left(\frac{B_{r}}{B_{z}}\right)^{\prime}+m\left[(2 m-1)\left(\frac{r_{o}^{\prime}}{r_{o}}\right)^{2}+\frac{r_{o}^{\prime \prime}}{r_{o}}\right]\right\} \frac{B_{z} v_{r}}{r_{o}^{m-1}}=0
\end{aligned}
$$

where operator ${ }^{\prime} \equiv \mathrm{d} / \mathrm{d} z, \omega$ is the angular frequency, $m$ is the azimuthal wave number (a positive integer) and

$$
c_{k}^{2}(z)=\frac{2 B_{z}^{2}(z)}{\mu\left(\rho_{i}(z)+\rho_{e}(z)\right)}
$$

is the fast kink speed. In the case of constant $B_{z}$, Eq. (5.1) simply reduces to

$$
\frac{\mathrm{d}^{2} v_{r}}{\mathrm{~d} z^{2}}+\left(\frac{\omega}{c_{k}}\right)^{2} v_{r}=0
$$

which is independent of $m$, in agreement with the results of Dymova \& Ruderman (2005) and Erdélyi \& Verth (2007). Analytical solutions to Eq. (5.3) have been extensively studied by Dymova \& Ruderman (2005), Erdélyi \& Verth (2007) and Verth et al. (2007). Two possible observable signatures of density stratification are anti-node peak shift of the first harmonic towards the loop footpoints (see Verth et al. 2007) and frequency ratio of the first harmonic and the fundamental mode, $\omega_{2} / \omega_{1}$ being less than 2 (see e.g., Andries et al. 2005a).

When there is magnetic stratification for the observed fast kink mode $(m=1)$, Eq. (5.1) becomes

$$
\begin{aligned}
& \left(B_{z} v_{r}\right)^{\prime \prime}+\frac{1}{2 r_{o}}\left(\frac{B_{r}}{B_{z}}+4 r_{o}^{\prime}\right)\left(B_{z} v_{r}\right)^{\prime} \\
& +\left[\left(\frac{\omega}{c_{k}}\right)^{2}+\frac{1}{2 r_{o}}\left(\frac{B_{r}}{B_{z}}\right)^{\prime}+\left(\frac{r_{o}^{\prime}}{r_{o}}\right)^{2}+\frac{r_{o}^{\prime \prime}}{r_{o}}\right] B_{z} v_{r}=0 .
\end{aligned}
$$

Assuming constant densities, $\rho_{e}$ and $\rho_{i}$ and using the explicit expressions for equilibrium quantities from Eqs. (3.10), (3.11) and (3.12), Eq. (5.4) is equivalent to

$$
\begin{aligned}
& {\left[a_{1} \cosh ^{2}\left(\frac{z}{L}\right)+a_{2} \cosh \left(\frac{z}{L}\right)+a_{3}\right] v_{r}^{\prime \prime}+\sinh \left(\frac{z}{L}\right)\left[a_{4} \cosh \left(\frac{z}{L}\right)+a_{5}\right] v_{r}^{\prime}} \\
& \quad+\left[a_{6} \cosh ^{2}\left(\frac{z}{L}\right)+a_{7} \cosh \left(\frac{z}{L}\right)+a_{8}+a_{9} \omega^{2}\right] v_{r}=0
\end{aligned}
$$

where $a_{n}$ are constants. Unfortunately, we know of no analytical solution to equations of general type Eq. (5.5). However, Eq. (5.5) is trivial to solve numerically by e.g., the shooting method. Solving Eq. (5.5) for the fundamental mode and first harmonic, the observable signatures of magnetic stratification are plotted in Fig. 2 for $\Gamma \in[1,2]$. In contrast to the case of density stratification with constant magnetic field (see Verth et al. 2007), the anti-node shift of the first harmonic is towards the loop apex (see Fig. 2a). 

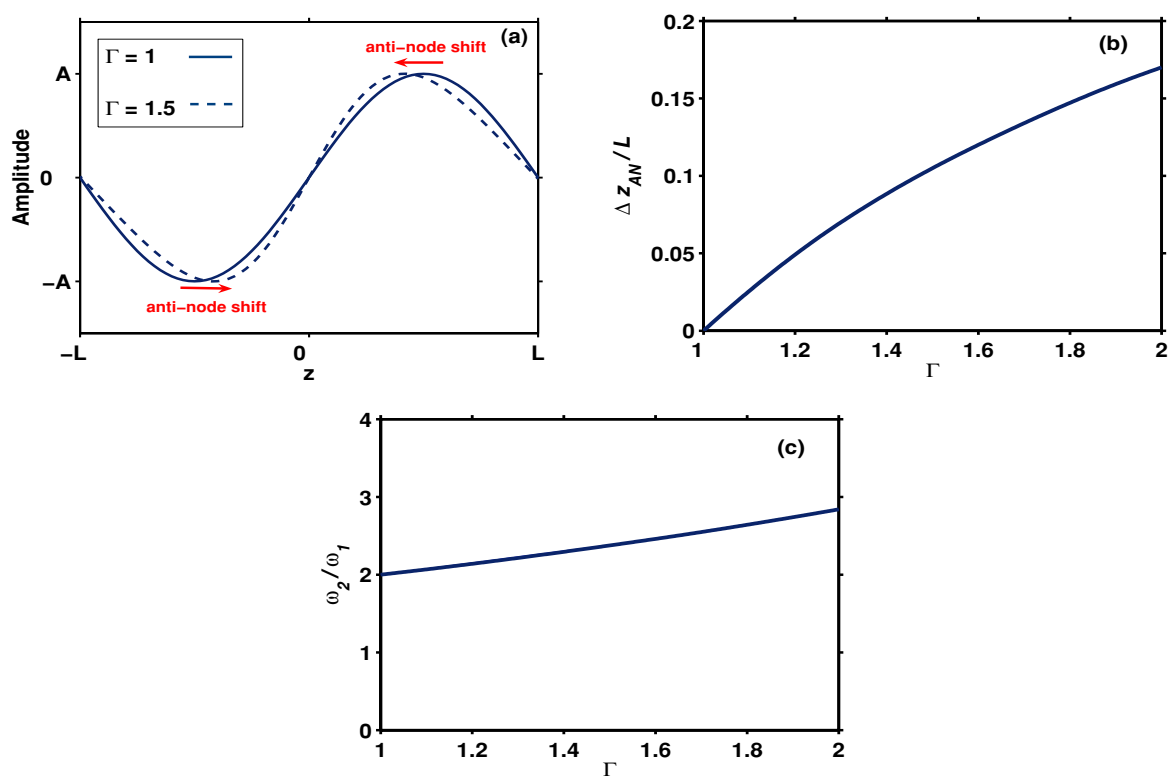

Figure 2. (a) Comparison of 1st harmonic amplitude profiles with constant magnetic field $(\Gamma=1)$ and magnetic stratification $(\Gamma=1.5)$. (b) Anti-node shift $\Delta z_{A N} / L$ for 1st harmonic plotted against $\Gamma$. (c) Frequency ratio of 1 st harmonic and fundamental mode $\omega_{2} / \omega_{1}$ against $\Gamma$.

The normalised anti-node shift of the first harmonic, $\Delta z_{A N} / L$ is plotted against $\Gamma$ in Fig. 2b. Note that for $\Gamma$ approximately less than 1.5 there is almost a linear relationship with $\Delta z_{A N} / L$. In further contrast to the case of density stratification with constant magnetic field (see Andries et al. 2005a), the frequency ratio of the first harmonic to the fundamental mode, $\omega_{2} / \omega_{1}$ is greater than 2 (see Fig. 2c).

There have been various studies to calculate the value of $\Gamma$ for coronal loops in both soft X-ray and EUV. Using Yohkoh data, Klimchuk (2000) found that the mean value of $\Gamma$ for a sample of 43 soft X-ray loops was 1.30. In another study using EUV TRACE data, Watko \& Klimchuk (2000) found that the mean $\Gamma$ value for post-flare loops was 1.13. However there may have been large uncertainties in these results since loop width was often at the resolution threshold of these instruments. Errors could also have been introduced by e.g., incorrect background subtraction and line of sight effects. Even allowing for a relatively small expansion factor of $\Gamma=1.13$, this should give measurable observable effects. E.g., a loop half length $L=100 \mathrm{Mm}$ and fundamental mode period 5 minutes, $\Gamma=1.13$ will give an anti-node shift of $3.5 \mathrm{Mm}$ and a change in the period of the first harmonic of -6.23 seconds. Certainly, spatial changes to the amplitude profile of a few $\mathrm{Mm}$ is within the current resolution of TRACE. Measuring changes in frequency down to the order of seconds may be possible with the fastest time cadences of the planned EUV imagers onboard $S D O$ and $S O$ (signal to noise ratio permitting).

Physically, it is reasonable to expect that both the plasma density and magnetic field strength decrease from the footpoints of a coronal loop towards its apex. If we observe a loop oscillating with $\omega_{2} / \omega_{1}<2$ then density stratification is likely to be the prevailing factor and if $\omega_{2} / \omega_{1}>2$ then magnetic field divergence is probably dominating. Quantifying the relative contribution from is each effect is more problematic.

To illustrate this, suppose that a semi-circular coronal loop has an exponentially density stratification with scale height $H$. Let us assume that both the density and magnetic 

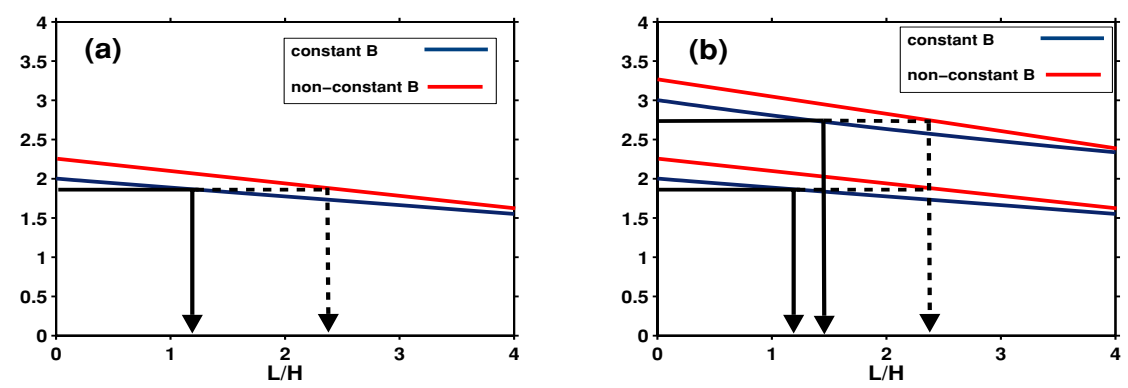

Figure 3. (a) Plot showing how a non-constant magnetic field affects the measurement of $L / H$ from the ratio $\omega_{2} / \omega_{1}$. (b) Plot showing how a non-constant magnetic field affects the measurement of $L / H$ using the ratios of both $\omega_{2} / \omega_{1}$ and $\omega_{3} / \omega_{1}$.

field profiles are non-constant $(L / H>0$ and $\Gamma>1)$ and that density stratification is the dominant effect so that $\omega_{2} / \omega_{1}<2$. If we want to measure $L / H$ from the observed value of $\omega_{2} / \omega_{1}$ and wrongly assume that the magnetic field is constant then $L / H$ will be underestimated (see Figure 3a). If the frequency of another mode, e.g., $\omega_{3}$ is also measured and the ratios of $\omega_{2} / \omega_{1}$ and $\omega_{3} / \omega_{1}$ are plotted against $L / H$ it is apparent that assuming a constant magnetic field results in two inconsistent values for $L / H$ (see Figure $3 \mathrm{~b})$. However, there will be a unique value of $\Gamma$ that results in two consistent values for $L / H$.

\section{Conclusions}

Hitherto, the magneto-seismological technique of using observations of the fast kink body mode to determine the density scale height of coronal loops has neglected to take into account corrections due to magnetic stratification. The MHD wave theory presented here shows that to carry out more precise magneto-seismology one must accurately quantify the effect of magnetic stratification on the amplitude profile and/or frequency of loop oscillations. This is problematic since, as mentioned previously, the precise fine structure of the magnetic field along coronal loops is not well understood at this time. It is hoped that Hinode will give a us greater understanding of the structure of the magnetic field in the photosphere, since for first time we will be able to measure the full magnetic field vector. This in turn should lead to more accurate nonlinear force-free magnetic field extrapolations from which we will better be able to determine the magnetic field structure along coronal loops.

Although, the magnetic field for the model presented in this paper has a relatively simple force-free (potential) structure, this maybe a reasonable approximation for many active region loops. E.g., extrapolations from the magnetogram data of the predominantly dipolar active region (AR 8270) where the first transversal loop oscillations were observed using TRACE (14th July 1998) show that magnetic field is approximately potential (see e.g., Ofman 2007). Therefore, the results described in Sect. 5 could be employed as a valuable new magneto-seismological tool to complement both emission measure and magnetic field extrapolation studies of suitable active regions. This in turn, could ultimately provide us with a more complete understanding of plasma fine structure in the solar corona.

\section{Acknowledgements}

G.V. is grateful to the Engineering and Physical Sciences Research Council (EPSRC), UK for the financial support received. 


\section{References}

Andries, J., Arregui, I., \& Goossens, M. 2005a, ApJ (Letters) 624, L57

Andries, J., Goossens, M., Hollweg, J. V., Arregui, I., \& Van Doorsselaere, T. 2005b, A\& $A$ 430, 1109

Arregui, I., Andries, J., Van Doorsselaere, T., Goossens, M., \& Poedts, S. 2007, A\&A 463, 333

Arregui, I., Van Doorsselaere, T., Andries, J., Goossens M., \& Kimpe, D. 2006, Phil. Trans. R. Soc. A 384, 529

Aschwanden, M. J., De Pontieu, B., Schrijver, C. J., \& Title, A. M. 2002, Solar Phys. 206, 99

Aschwanden, M. J., Fletcher, L., Schrijver, C. J., \& Alexander, D. 1999a, ApJ 520, 880

Aschwanden, M. J., Newmark, J. S., Delaboudinière, J., Neupert, W. N., Klimchuk, J. A., et al. 1999b, Apj 515, 84

Aschwanden, M. J., Nightingale, R. W., \& Alexander, D. 2000, ApJ 541, 1059

Aschwanden, M. J., Nightingale, R. W., Andries, J., Goossens, M., \& Van Doorsselaere, T. 2003, ApJ 598, 1375

Aschwanden, M. J., Schrijver, C. J., \& Alexander, D. 2001, ApJ 550, 1036

Banerjee, D., Erdélyi, R., Oliver, R. \& O'Shea, E. 2007, Solar Phys. 246, 3

Brekke, P., Kjeldseth-Moe, O., \& Harrison, R. A. 1997, Solar Phys. 175, 511

Browning, P. K., \& Priest, E. R. 1982, Geophys. Ap. Fluid Dyn. 21, 237

Carter, B., \& Erdélyi, R. 2007, A\&A 475, 323

De Pontieu, B., \& Erdélyi, R. 2006, Phil Trans. Roy. Soc. A. 364, 383

Díaz, A.J., Oliver, R., \& Ballester, J.L. 2002, ApJ 580, 550

Dymova, M. V., \& Ruderman, M. S. 2005, Solar Phys. 229, 79

Edwin, P. M., \& Roberts, B. 1983, Solar Phys. 88, 179

Erdélyi, R. 2006a, Phil. Trans. Roy. Soc. A. 364, 351

Erdélyi, R. 2006b in: K. Fletcher (ed.) \& M. Thompson (sci. ed.), SOHO 18/GONG 2006/HELAS I, Beyond the spherical Sun, ESA SP-624, p15.1

Erdélyi, R., \& Carter, B. 2006, A\&A 455, 361

Erdélyi, R., \& Fedun, V. 2006, Solar Phys. 238, 41

Erdélyi, R., \& Fedun, V. 2007, Solar Phys. 246, 101

Erdélyi, R., \& Verth, G. 2007, A\&SA 462, 743

Goossens, M., Andries, J., \& Aschwanden, M. J. 2002, A\&A 394, L39-42

Goossens, M., Arregui, I., \& Andries, J. 2006, Phil. Trans. R. Soc. A. 364, 433

Jess, D. B., Mathioudakis, M., Erdélyi, R., Verth, G., McAteer, R. T. J., \& Keenan, F. P. 2007 Apj (in press)

Joarder, P. S., Nakariakov, V. M., \& Roberts, B. 1997, Solar Phys. 176, 285

Joarder, P. S., \& Narayanan, A. S. 2000, A\&A 359, 1211

Klimchuk, J. A. 2000, Solar Phys. 193, 53

Klimchuk, J. A., Antiochos, S. K., \& Norton, D. 2000, ApJ 542, 504

López Fuentes, M. C., Klimchuk, J. A., \& Démoulin, P. 2006, Apj 639, 459

McEwan, M. P., Donnelly, G. R., Díaz, A. J., \& Roberts, B. 2006, A $\& A 460893$

Mendoza-Bricẽno, C. A., Erdélyi, R., \& Sigalotti, L. Di G. 2004, ApJ 605, 493

Nakariakov, V.M., Ofman, L., DeLuca, E. E., Roberts, B., \& Davila, J. M. 1999, Science 285, 862

Nakariakov, V. M., \& Roberts, B. 1995, Solar Phys. 159, 213

Narayanan, A. S. 1991, Plasma Phys. Control. Fusion 33, 333

Ofman, L. 2007, ApJ 655, 1134

Ofman, L., \& Wang, T. 2002, ApJ (Letters) 530, L85

Ruderman, M. S. 2007, Solar Phys., 246, 119

Ruderman, M. S., \& Roberts, B. 2002, ApJ 577, 475

Schrijver, C. J. 2001, Solar Phys. 198, 325

Somasundaram, K., Venkatraman, S., \& Sengottuvel, M. P. 1999, Plasma Phys. Control. Fusion 41, 1421

Terra-Homem, M., Erdélyi, R., \& Ballai, I. 2003, Solar Phys. 217, 199

Tirry, W. J., Cadez, W. M., Erdélyi, R., \& Goossens, M. 1998, A $\& A$ 332, 786 
Van Doorsselaere, T., Andries, J., Poedts, S., \& Goossens, M. 2004, ApJ 606, 1223

Verwichte, E., Nakariakov, V. M., Ofman, L., \& Deluca, E. E. 2004, Solar Phys. 223, 77

Verth, G. 2007, Astron. Nachr. 328, 746

Verth, G., \& Erdélyi, R. 2007 in: E. Marsch, K. Tsinganos, R. Marsden \& L. Conroy (eds.), The $2^{\text {nd }}$ Solar Orbiter Workshop, ESA SP-641

Verth, G, Van Doorsselaere, T., Erdélyi, R., \& Goossens, M. 2007, A\& A 475, 341

Watko, J.A., \& Klimchuk, J. A. 2000, Solar Phys. 193, 77

Winebarger, A. R., DeLuca, E. E., \& Golub, L. 2001, ApJ 553, L81

Winebarger, A. R., Warren, H., van Ballegooijen, A., DeLuca, E. E., \& Golub, L. 2002, ApJ $567, \mathrm{~L} 89$

Winebarger, A. R., Warren, H. P., \& Seaton, D. B. 2003, ApJ 593, 1164 